\title{
TOOL CONDITION MONITORING AND TOOL DEFECT DETECTION FOR END MILLS BASED ON HIGH-FREQUENCY MACHINE TOOL DATA
}

\author{
Alexander Fertig ${ }^{1,{ }^{*}}$, Lukas Grau, Marius Altmannsberger, Matthias Weigold ${ }^{1}$
}

\author{
${ }^{1}$ Institute for Production Management, Technology and Machine Tools (PTW), Otto-Berndt-Straße 2, 64287 Darmstadt, Germany
} ${ }^{*}$ Corresponding a.fertig@ptw.tu-darmstadt.de

\begin{abstract}
In the context of increasing digitalization, machine tools have a decisive impact on the manufacturing of technically sophisticated products. The resulting large amount of available data opens up new opportunities for process monitoring and optimization. In this paper, a new in-process tool condition monitoring (TCM) approach for end mills is developed. Besides in-process wear determination, the presented approach also enables the early detection of tool manufacturing defects on end mills. By applying machine learning algorithms, high prediction accuracies can be achieved. The results allow the implementation of an in-process TCM system based on internal machine tool data.
\end{abstract}

Keywords:

Tool Condition Monitoring; Machine Learning; Defect classification; Internal machine tool data

\section{INTRODUCTION}

New technologies enable in the context of increasing digitalization ever more precise production with increased product quality. Particularly the production of components made of difficult-to-cut materials for the aerospace industry is facing high challenges in terms of economic efficiency and productivity in an increasingly globalized market environment. [Pfeifer, 2006] One strategy for companies to address these requirements is to optimize the costs of machining. Here, the focus is mostly on the tool as a cost driver and essential factor for product quality. [FrancoGasca, 2006] To fulfill these objectives additional knowledge can be generated based on the increasing availability of data from production processes that enable data-driven decisions. [Bergs, 2020] In this context, tool condition monitoring (TCM) aims to provide information on the actual and prospective tool condition by gathering machine-related data in parallel to the process. The successful implementation of a TCM System enables not only the optimized utilization of tool life but also a reduction in the use of resources and an increase in product quality. [Wang, 2013; Zhu, 2009] This paper presents a new approach for TCM based on high-frequently recorded internal machine tool data. In this context, a finishing process using end mills is used to show how the current tool wear can be determined based on machine learning (ML) methods. In addition, it is demonstrated how production errors during tool production affect the machine tool data. These errors are subsequently detected by the TCM system using a machine learning based classification approach.

\section{STATE OF THE ART}

In the field of TCM, there are many research publications and approaches. The literature reviews submitted by [Jantunen, 2002], [Rehorn, 2005], [Zhu, 2009], [Teti, 2010], and [Lauro, 2014] serve to provide an overview in this large field of research. [Jantunen, 2002] introduces TCM approaches for turning processes. As a result, cutting forces and torques are the most frequently considered signals. Signal processing focuses on the use of the time domain features of the signals. Spectral analyses with Fast Fourier Transforms (FFT) commonly accompany signal processing. [Rehorn, 2005] show that many approaches to tool monitoring, especially for the inspection of end mills, are based on the use of cutting forces. In addition, the authors point out that for the application of a TCM system in the industry, its simplicity is of primary importance. [Zhu, 2009] distinguish between the indirect methods for determining the tool condition, the measurement of cutting forces, vibrations, sound emissions, currents, and image data. In addition to the methods mentioned above, analyses in the time-frequency domain, such as wavelet transforms (WT), are used for feature generation and noise reduction. In their summary of the various sensors used, [Teti, 2010] provide an overview of the possibilities that exist for TCM. The power and currents of the drive motors, forces, torques, acoustic emissions, and vibrations are addressed. Based on these sensor signals, feature extraction methods are used in the time domain, frequency domain, and timefrequency domain. In addition to conventional statistical analysis, Principal Component Analysis (PCA) for dimension reduction plays an important role in the time 
domain. Addressing the frequency domain, the FFT dominates, which is extended to the STFT - Short Time Fourier Transform for the temporal resolution of the frequencies. WT and Hilbert-Huang-Transform (HHT) offer further methods in the time-frequency domain. [Lauro, 2014] present similar scenarios in their publication and distinguish five basic monitoring techniques based on the measurement of cutting forces, vibrations, temperatures, acoustic emissions, and sound. For signal processing, FFT, WT, and HHT are examined in the publications, following [Teti, 2010]. In addition to these summarizing studies, further developments in TCM can also be seen. [Benkedjouh, 2018] use blind source separation and WT on externally applied accelerometers and force signals to separate relevant from irrelevant signal components. The subsequent feature extraction is performed based on the force signals. Further research on TCM for end milling is presented by [Binsaeid, 2009], [Chen, 2005], [Grasso, 2013], [Li, 2017], [Lin, 2017], [Sevilla-Camacho, 2011] and [Niu, 2020]. In addition to the already mentioned approaches based on external sensors, these also include approaches that use the already installed machine tool sensors to provide the necessary signals for TCM. In these, mainly internal currents of the drives are used for TCM. It can be concluded that all presented approaches provide the basis for the implementation of a TCM system. However, most of the approaches are associated with high installation costs for external sensors, which is why they are not suitable for broad application. The first approaches using internal machine tool sensors only focus on currents and do not consider other promising signals such as position data and control deviations.

In addition to tool wear, this paper aims to detect defects that occur during tool manufacturing. The focus is on geometry-changing defects of the tool. The literature provides some studies for this purpose, which investigate the influences of changed tool geometries on the cutting forces, the surface of the manufactured workpiece, and tool wear propagation. [Westermann, 2015] investigate the variation of tool orthogonal rake angle, tool orthogonal clearance, and cutting edge roundings on tool wear. They observe that the considered variations of the tool geometry have an influence on tool wear and the cutting performance changes depending on the amount of variation. [Conradie, 2015] conclude that an increased tool orthogonal clearance can reduce flank wear. [Suresh Kumar Reddy, 2005] describe that tool orthogonal rake angles between $4^{\circ}$ and $10^{\circ}$ of end mills produce the best surface quality within their milling tests of AISI 1045 steel. [Muhammad Syafik, 2017], [Kalidass, 2014], [Izamshah, 2013], [Wan, 2017], [Ema, 1989] provide works investigating the influence of the helix angle on tool wear and resulting cutting forces. The results illustrate that a change in the helix angle leads to a change in the cutting forces. Accordingly, these effects should also be detectable in the internal machine data. Studies on different tool lifetimes as a result of changed cutting edge rounding are obtained from [Westermann, 2015] and [Krain, 2007].

Based on the presented state of the art, this paper aims to investigate the influence of the mentioned changes in geometry characteristics of the end mill on internal drive signals. The goal is to detect them during the application of the tool. The main objective is to create the basis for a TCM system that detects the condition of the tool in terms of wear and defect-free geometry. This monitoring enables faulty processes to be reduced and the quality to be increased.

\section{EXPERIMENTAL DESIGN \& METHODOLOGY}

\subsection{Experimental design}

The basis of the investigation consists of a linear end milling process with end mills of $8 \mathrm{~mm}$ diameter and 4 cutting edges, which were supplied by Linner $\mathrm{GmbH}$. The material used for the test series is the titanium alloy Ti6Al4V, which is often used for the production of aerospace components [M'Saoubi, 2015]. The tests were conducted on the vertical machining center DMG MORI DMC 850V equipped with a Siemens Sinumerik 840D sl control unit. During the milling operations, the internal machine tool data is recorded and processed with an edge-computing solution, which provides data at sampling rates of $500 \mathrm{~Hz}$. The recorded internal signals are summarized in Tab. 1, with $X$ and $Y$ as the horizontal feed axes an $Z$ as the vertical spindle axis. In addition, the NC code block has been recorded for the contextualization of the data. A Keyence VHX 5000 microscope is used to measure the flank wear as the main deterioration phenomenon of tool wear. To attain a high accuracy of the direct wear measurements at high efficiency, the measurement intervals were adapted to the three phases of initial wear, linear wear, and progressive wear.

Tab. 1: Recorded machine tool signals

\begin{tabular}{lc}
\hline \multicolumn{1}{c}{ Signal } & Axis \\
\hline $\begin{array}{lc}\text { Current, encoder position, control } \\
\text { position, commanded position, }\end{array}$ \\
$\begin{array}{lc}\text { desired position, commanded speed, } \\
\text { control difference }\end{array}$ & $\mathrm{X}, \mathrm{Y}, \mathrm{Z}$, Spindle \\
Contour deviation & \\
Power, torque & $\mathrm{X}, \mathrm{Y}, \mathrm{Z}$ \\
\hline
\end{tabular}

Tab. 2 shows the experimental design with different end mills and technology parameters. Defect-free tools are named reference tools (RT). Tools with manufactured defects, thus with geometrical deviations, are named error tools (ET). The ET were specifically provided with production errors that influence the geometric properties of the end mills. The resulting deviations, which are shown in Tab. 3, result in 14 target classes for the subsequent classification task

Tab. 2: Cutting parameter variations for the linear milling processes. $v_{c}=$ cutting speed; $f_{z}=$ feed per tooth, $a_{e}=$ width of cut, $a_{p}=$ depth of cut

\begin{tabular}{ccccc}
\hline Tool & $\begin{array}{c}\mathbf{v}_{\mathbf{c}} \text { in } \\
\mathbf{m} / \mathbf{m i n}\end{array}$ & $\begin{array}{c}\mathbf{f}_{\mathbf{z}} \text { in } \\
\mathbf{m m}\end{array}$ & $\begin{array}{c}\mathbf{a}_{\mathrm{e}} \text { in } \\
\mathbf{m m}\end{array}$ & $\begin{array}{c}\mathbf{a}_{\mathbf{p}} \text { in } \\
\mathbf{m m}\end{array}$ \\
\hline $\begin{array}{c}\text { Reference tools } \\
\text { (RT) }\end{array}$ & 50,80, & 0.05, & 0.8, & 8 \\
Error tools (ET) & 100 & 0.025 & 1.6 & \\
\hline
\end{tabular}

Tab. 3 : Resulting deviations on the error tools. The characteristics of the reference tools are marked with (reference) 


\begin{tabular}{lll}
\hline $\begin{array}{l}\text { Resulting error / influenced } \\
\text { geometric attribute }\end{array}$ & Characteristics & ET class (cf. Fig. 4) \\
\hline Helix angle & $40^{\circ}$ (reference, varies slightly from tooth to tooth); & $\mathrm{RT}, 1,2,3,13$ \\
& $30^{\circ} ; 50^{\circ}, 40^{\circ}$ (without variation from tooth to tooth), $40^{\circ}$ (even pitch) & \\
Rounding cutting edge & $8 \mu \mathrm{m}$ (reference); $16 \mu \mathrm{m}$ & $\mathrm{RT}, 4$ \\
Tool orthogonal rake angle & $12^{\circ}$ (reference); $5^{\circ} ; 2^{\circ}$ & $\mathrm{RT}, 5,6$ \\
Tool orthogonal clearance & $14^{\circ}$ (reference); $7^{\circ}$ & $\mathrm{RT}, 7$ \\
Cutting edge radius (for one & $4 \mathrm{~mm}$ (reference); $3.7 \mathrm{~mm}$ & $\mathrm{RT}, 8$ \\
cutting edge only) & & $\mathrm{RT}, 9$ \\
Radial deviation & $<10 \mu \mathrm{m}$ (reference); $40 \mu \mathrm{m}$ & $\mathrm{RT}, 10$ \\
Web thickness & $4.1 \mathrm{~mm}$ (reference); $5.1 \mathrm{~mm}$ & $\mathrm{RT}, 11$ \\
Coating & coated (reference); uncoated & $\mathrm{RT}, 12$ \\
Grain size & reference grain size; larger grain size & \\
\hline
\end{tabular}

\subsection{Methodology - Knowledge Discovery in Databases}

Three key approaches for this study are distinguished in the scientific literature: Knowledge Discovery in Databases (KDD), Cross-Industry Standard Process for Data Mining (CRISP-DM), and SEMMA - Sample, Explore, Modify, Model, and Assess. While KDD is the original concept developed for scientific purposes, the other two are adaptations for industry-focused usage. [Fayyad, 1997; Shafique, 2014; Wirth, 2000] The comparison of these concepts highlights KDD as the most suitable methodology within this study, mainly due to reproducibility and high acceptance in science. Fig. 1 depicts the key steps, which will be used as the framework for the following chapters of this elaboration.

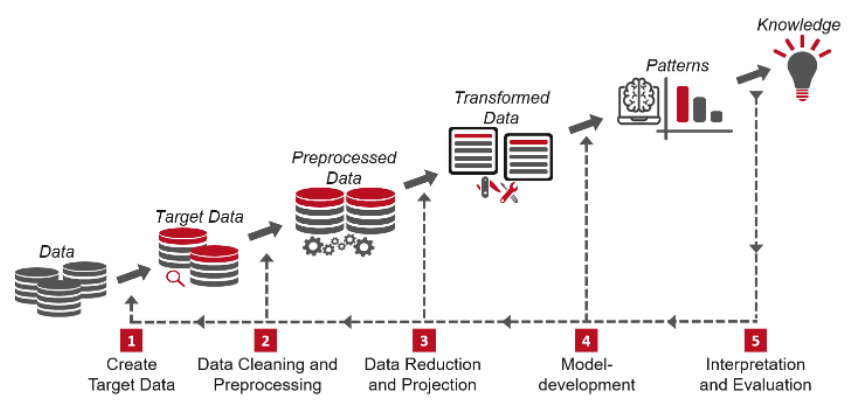

Fig. 1: The KDD Process (own representation according to [Fayyad, 1996])

\section{DATA ANALYSIS}

After the completed raw data acquisition, data analysis according to the KDD can be conducted with the following steps: Create Target Data, Data Cleaning and Preprocessing as well as Data Reduction and Projection. In the further course, internal machine tool data and wear data will be distinguished.

\subsection{KDD Steps 1 and 2: Create Target Data, Data Cleaning, and Preprocessing}

To create a target dataset from the recorded raw machine tool data, structural mistakes, as well as wrong data, were removed. Furthermore, signals containing no or only redundant information were eliminated. After the following optimization of data types, the last remaining inconsistencies were detected and debugged. The data is available in individual data slices by using triggers in the NC
Raw data slices - contains data without tooth engagement

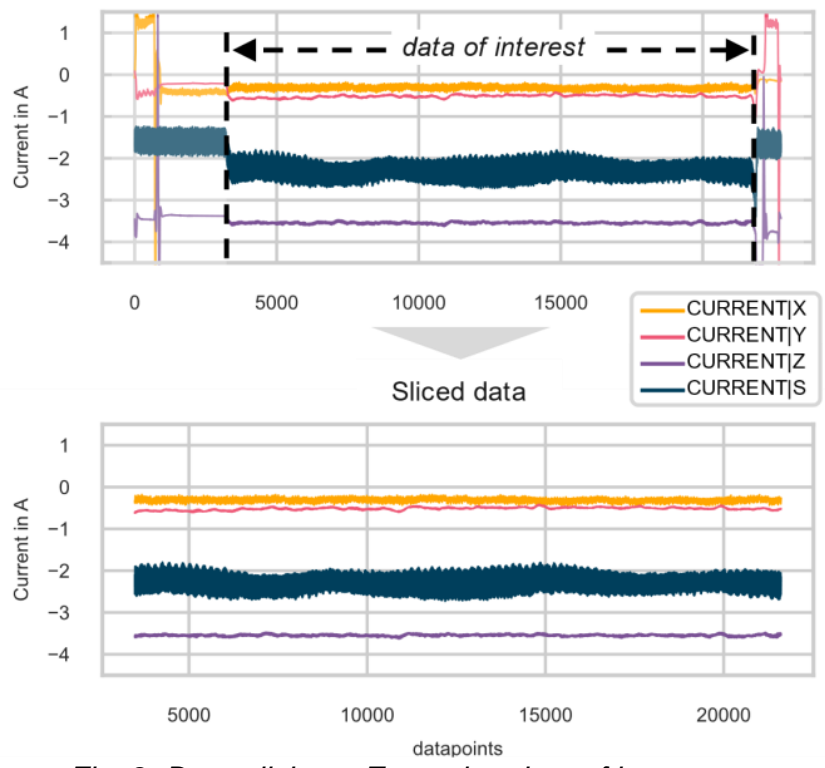

Fig. 2: Data-slicing - Extracting data of interest

code. Each slice contains the data from one process. As shown in Fig. 2, these data must be reduced to the data of interest - i.e. the data during which tooth engagement persists - for the analysis. This elimination of unnecessary data ensures that only data generated during tooth engagement is used for the subsequent analysis. This step was implemented automatically using the position signals. By knowing the process flow, it was possible to extract the data of interest automatically. To label the machine tool data appropriately, the raw wear data has to be prepared as well. The key factor at this point is the interpolation of wear data so that one label can be allocated to every milling process thus the time-series segment of the internal signals. The interpolation methods examined in this study include the following: linear, cubic spline, monotonic cubic (PCHIP), piecewise cubic (Akima), piecewise polynomial (Bernstein basis), and polynomial (Krogh), which were calculated using the Python library scipy. [Virtanen, 2020] The conducted evaluation results in the use of the Akima interpolation method, which results in best fitting wear curves. Fig 3 shows one recorded wear curve and its interpolation. 


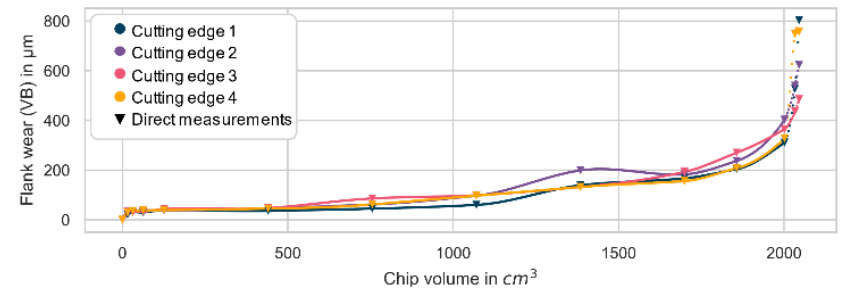

Fig 3: Wear curve for a reference cutter showing measured and interpolated values based on Akima interpolation

\subsection{KDD Step 3: Data Reduction and Projection}

Having a clean, labeled dataset the variables can be plotted over flank wear. This allows a first visual qualitative data evaluation and reduction step. As faulty data was already eliminated and hidden patterns might be present, all resulting signals were used for data projection. Data projection comprises the application of features to the set of signals, often also referred to as feature engineering. The result of the conducted literature research is a portfolio of features that are used further on. These are divided into features in the time domain, frequency domain, and timefrequency domain. The calculated time domain features are: Median, Mean, Median absolute deviation, Mean absolute deviation, Minimum, Maximum, Positive turning points, Negative turning points, Neighborhood peaks, Root mean square, Skewness, Kurtosis, Centroid, Slope, Variance, Auto-Correlation, and Entropy. For the frequency-domain following features were used: Fundamental frequency, Maximum frequency, Median frequency, Minimum frequency, Power bandwidth, Peak to peak distance, Spectral decrease, Spectral slope, Spectral centroid, Spectral distance, Spectral entropy, Spectra spread, Spectral roll-on, Spectral roll-off, Spectral skewness, Spectral kurtosis, Spectral variation, and Max power spectrum. For the time-frequency domain, the wavelet entropy serves for further analyses. All features were calculated by using the Python library TSFEL [Barandas, 2020] for window sizes of 500, 1000, and 2000 data points to determine how large the window size needs to be to prevent losing relevant information. This was necessary to estimate the potential response time of the intended TCM-System. After generating the features, all 481 features were plotted and analyzed qualitatively. The evaluation of the plots confirms the use of a feature window with 1000 data points, which is a trade-off between signal quality and quantity. In total, this results in 77090 samples of labeled real data for the following model development. In addition, the first analysis shows degressive curves in the consideration of the basic statistical features (mean, std. etc.) for the drive currents for all axis directions. Furthermore, the features spectral skewness and spectral kurtosis show promising trajectories when tool wear is increasing. In the consideration of the ET plots, some features show level differences between the different tools. This indicates a good separation of RT and ET. This applies independently from the selection of the cutting parameters. This manual qualitative feature understanding process serves as the basis for the further feature selection process. Therefore, different feature selection algorithms based on filter (pearson-correlation, chi-square test, F-regression), wrapper (recursive-feature-elimination), and hybrid (LASSO, lightGBM) algorithms were applied to identify the most relevant features for the subsequent machine learning based modeling. The results of each algorithm are ranked according to the relevance of the respective feature. Within the tables containing the ranked features, the absolute frequency of the occurrence for each feature is counted. This results in the following top 20 features for the classification task: signal distance, negative turning points, mean absolute difference, mean absolute deviation and max power spectrum of Current|X, signal distance, neighborhood peaks, negative turning points and mean absolute difference of Current|Y, root mean square, mean, min of Current|Z, wavelet entropy of control difference signals of the $\mathrm{Y}$-axis were selected for the subsequent modeling. For regression, the spectral roll-off, root mean square, power bandwidth, positive turning points, negative turning points median, and mean of Current|X and spectral distance, root mean square, min, median, and mean of Current|Y, and spectral spread, spectral slope, sprectral skewness, spectral kurtosis, spectral centroid, power bandwidth, positive turning points, negative turning points of Encoder_position|Z were determined as top 20 useful features for the regression task.

\section{MODELING}

For a better understanding, steps 4 and 5 of the KDD process are presented in this chapter.

\subsection{KDD Step 4: Model development}

After feature selection, the most important features are fitted into different machine learning models. To select promising algorithms an exploratory analysis of the resulting features using pairplots was performed. Fig. 4 shows a representative example of a pairplot in which different features were compared to each other in two dimensions to indicate possibilities for the separation of the target classes.

\section{Classification Algorithms}

Considering the data distribution, it can be assumed that geometric ML algorithms can achieve good classification accuracies. The data in the pair plots are distributed in contiguous point clouds, which can already be delimited with elliptical borders. Therefore, a k-nearest-neighbor algorithm (KNN) should deliver good classification results. In addition, a support vector machine (SVM) that separates the data with the help of different hyperplanes seems to be promising. However, since the data often overlap at the edges, an exact separation of the features into target classes with SVM seems not always possible. In addition, a random forest (RF) algorithm is used.

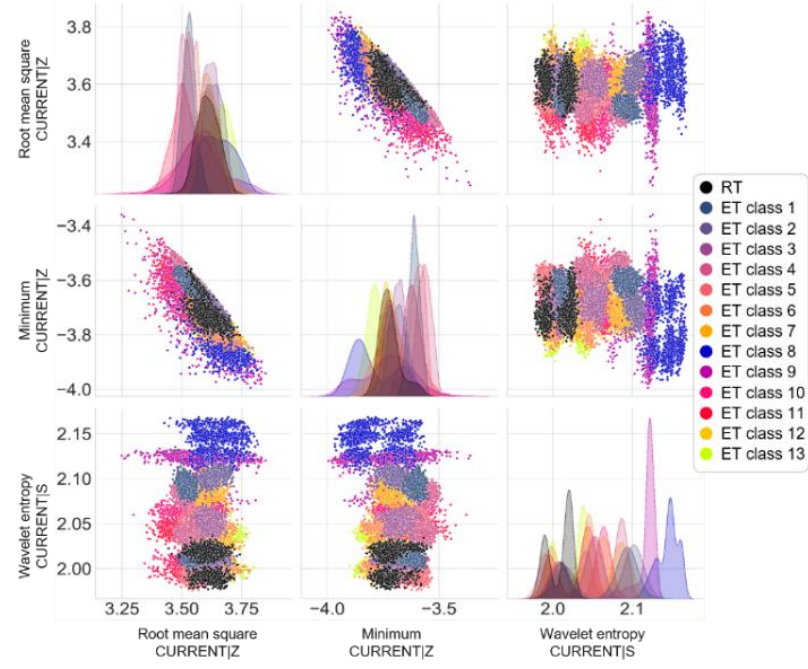

Fig. 4: Pairplot for three selected features. RT represents the reference tool class, ET represent the error tool classes 
The following metrics are used for the evaluation of the prediction:

$$
\begin{aligned}
& \text { Accuracy }=\frac{T P+T N}{T P+T N+F P+F N} \\
& \text { Precision }=\frac{T P}{T P+F P} \\
& \text { Recall }=\frac{T P}{T P+F N}
\end{aligned}
$$

with $T P$ as true-positive, $T N$ as true-negative, $F P$ as falsepositive, and $F N$ as false-negative predictions. In this case, a TP prediction corresponds to a correct predicted class affiliation.

\section{Regression Algorithms}

For the regression of tool wear, linear regression, SVM, decision tree (DT), and random forest (RF) were identified as suitable models to be used within this study. Linear regression works well as a comparative model. The DT provides a method to learn suitable wear values for sufficiently small intervals of the feature values. The RF as an ensemble learning algorithm enables an estimation of the wear value by multihit decision of many generated decision trees. The selection of SVMs is based on its common application in the literature. For the regression, the metrics mean absolute error (MAE) and R-squared (R2) were used according to the following equations (cf. [Wu, 2017]):

$$
\begin{aligned}
& \operatorname{MAE}(y, \widehat{y})=\frac{1}{n_{\text {samples }}} \sum_{i=0}^{n_{\text {samples }}-1}\left|y_{i}-\widehat{y}_{i}\right| \\
& R^{2}(y, \widehat{y})=1-\frac{\sum_{i=1}^{n_{\text {samples }}}\left(y_{i}-\widehat{y}_{i}\right)^{2}}{\sum_{i=1}^{n_{\text {samples }}}\left(y_{i}-\bar{y}_{i}\right)^{2}}
\end{aligned}
$$

with $y_{i}$ as true value, $\hat{y}_{i}$ as predicted value, $\bar{y}_{i}$ as the mean of true values, and $n_{\text {samples }}$ as the number of samples in the test set.

\subsection{KDD Step 5: Results \& Discussion}

\section{Results for classification}

As shown in Tab. 4 the KNN was able to generate a high classification accuracy of $94.66 \%$ for tool defect classes. The low dispersion of the misclassifications is remarkable. Furthermore, the SVM only achieved a low classification accuracy of $39 \%$. As already mentioned, this is most likely due to the overlapping data points of the different classes, which could not be properly separated from the hyperplanes. The RF was able to generate the model with the best classification accuracy of $98 \%$. However, the assumption is that this is overfitting, as the accuracy of this algorithm on all training sets reached $100 \%$. By selecting the KNN algorithm for further statements it is worth mentioning that when looking at the recall for the class reference tool (when considering a binary classification with RT and ET as target class), it reaches $99.1 \%$. In this case, only three samples were predicted to not belong to the reference tool. The precision for this class achieved $100 \%$. These results show the potential for manufacturing error detection in milling tools during their use based on internal machine tool data.
Tab. 4: Results for tool defect classification

\begin{tabular}{lccc}
\hline \multicolumn{1}{c}{ Algorithm } & $\begin{array}{c}\text { Accuracy } \\
\text { test set }\end{array}$ & Precision & Recall \\
\hline $\begin{array}{l}\text { K-Nearest- } \\
\text { Neighbor }\end{array}$ & $94.49 \%$ & $94.47 \%$ & $94.43 \%$ \\
$\begin{array}{l}\text { Support Vector } \\
\text { Machine }\end{array}$ & $38.72 \%$ & $39.31 \%$ & $38.39 \%$ \\
$\begin{array}{l}\text { Random } \\
\text { Forest }\end{array}$ & $97.99 \%$ & $97.99 \%$ & $97.97 \%$ \\
\hline
\end{tabular}

\section{Results for regression}

Tab. 5 shows the mean absolute error (MAE) and Rsquared $\left(R^{2}\right)$ for the tool wear regression performed in this study. In this case, the decision tree performed best with an MAE of $10 \mu \mathrm{m}$. This low error for the wear prediction allows the conclusion that a sufficient prediction of the current condition of the tool is feasible for the wear as well. The other models are not able to represent the wear based on the data due to the significantly lower scores shown in Tab. 5. It should be mentioned, though, that at very high flank wear values from approx. VB $=300 \mu \mathrm{m}$ (in this case this means the tool is completely worn out), the predicted values become less accurate. This is due to the small number of samples gathered in this area. In addition, the progressive degradation of the tool plays a major role here, which leads to significantly more fluctuations during the process and thus in the data. Nevertheless, the reached accuracy allows an estimation of the remaining tool life under the current operating conditions during deployment. However, further tests on new data will still be needed to ensure the robustness of the developed approach.

Tab. 5: Results for tool wear regression

\begin{tabular}{lcc}
\hline \multicolumn{1}{c}{ Algorithm } & $\begin{array}{c}\text { MAE } \\
\text { test set }\end{array}$ & $\mathbf{R}^{\mathbf{2}}$ \\
\hline Linear Regression & $24 \mu \mathrm{m}$ & $65.33 \%$ \\
Support Vector Machine & $31 \mu \mathrm{m}$ & $41.05 \%$ \\
Decision Tree & $10 \mu \mathrm{m}$ & $85.77 \%$ \\
Random Forest & $30 \mu \mathrm{m}$ & $59.87 \%$ \\
\hline
\end{tabular}

\section{CONCLUSION \& OUTLOOK}

In this paper, a new approach that enables the condition of a tool to be determined during the milling process is presented. In addition to wear regression, production errors of the tools, which subsequently affect the cutting process, are also included as target variables. A finishing process with end mills was used for the investigation. The necessary data for the development of the data-driven approaches is provided by an edge computing solution that records and processes internal machine tool data at a frequency of $500 \mathrm{~Hz}$. The two underlying approaches, one for the classification of defective tools and the other for the regression of tool wear, show great potential for in-process TCM when looking at their high prediction accuracies. In further research, these initial principles will be used to build a robust TCM system for this process based on additional test series. The focus will be on the transferability of the developed approaches to other machining centers. Furthermore, the high scores imply that internal machine tool data, when recorded at high frequency, provide the necessary information with sufficient detail to apply datadriven models. This shows the great potential of high- 
frequency recorded internal machine tool data, which will be used for additional use cases in future work.

\section{ACKNOWLEDGMENTS}

This research and development project is funded by the German Federal Ministry of Education and Research (BMBF) within the "Innovations for Tomorrow's Production, Services, and Work" Program (funding number 02P17D123) and implemented by the Project Management Agency Karlsruhe (PTKA). The author is responsible for the content of this publication.

\section{REFERENCES}

[Barandas, 2020] Barandas, M., Folgado, D., Fernandes, L., Santos, S., Abreu, M., Bota, P., Liu, H., Schultz, T., Gamboa, H. TSFEL: Time Series Feature Extraction Library. SoftwareX 11, 2020, 100456.

[Benkedjouh, 2018] Benkedjouh, T., Zerhouni, N., Rechak, $\mathrm{S}$. Tool wear condition monitoring based on continuous wavelet transform and blind source separation. Int J Adv Manuf Technol 97, 2018, 33113323, 13 pp.

[Bergs, 2020] Bergs, T. Internet of Production - Turning Data into Value, 2020. Fraunhofer-Gesellschaft.

[Binsaeid, 2009] Binsaeid, S., Asfour, S., Cho, S., Onar, A. Machine ensemble approach for simultaneous detection of transient and gradual abnormalities in end milling using multisensor fusion. Journal of Materials Processing Technology 209, 2009, 4728-4738.

[Chen, 2005] Chen, J.C., Chen, J.C. An artificial-neuralnetworks-based in-process tool wear prediction system in milling operations. Int J Adv Manuf Technol 25, 2005, 427-434, 8 pp.

[Conradie, 2015] Conradie, P., Oosthuizen, T., Dimitrov, D., Saxer, M. Effect of milling strategy and tool geometry on machining cost when cutting titanium alloys. SAJIE 26, 2015.

[Ema, 1989] Ema, S., Davies, R. Cutting performance of end mills with different helix angles. International Journal of Machine Tools and Manufacture 29, 1989, 217-227.

[Fayyad, 1996] Fayyad, U., Piatetsky-Shapiro, G., Smyth, $P$. From Data Mining to Knowledge Discovery in Databases. Al Magazine 17, 1996, 37-54, 18 pp.

[Fayyad, 1997] Fayyad, U. Data mining and knowledge discovery in databases: implications for scientific databases: Proceedings : August 11-13, 1997 Olympia, Washington, 1997. IEEE Computer Society, Los Alamitos, CA, 2-11, 278 pp.

[Franco-Gasca, 2006] Franco-Gasca, L.A., Herrera-Ruiz, G., Peniche-Vera, R., Romero-Troncoso, R.d.J., LealTafolla, W. Sensorless tool failure monitoring system for drilling machines. International Journal of Machine Tools and Manufacture 46, 2006, 381-386.

[Grasso, 2013] Grasso, M., Albertelli, P., Colosimo, B.M. An Adaptive SPC Approach for Multi-sensor Fusion and Monitoring of Time-varying Processes. Procedia CIRP 12, 2013, 61-66, 6 pp.

[Izamshah, 2013] Izamshah, R., Yuhazri, M.Y., Hadzley, M., Ali, M.A., Subramonian, S. Effects of End Mill Helix Angle on Accuracy for Machining Thin-Rib Aerospace Component. AMM 315, 2013, 773-777.

[Jantunen, 2002] Jantunen, E. A summary of methods applied to tool condition monitoring in drilling.
International Journal of Machine Tools and Manufacture 42, 2002, 997-1010.

[Kalidass, 2014] Kalidass, S., Palanisamy, P Experimental Investigation on the Effect of Tool Geometry and Cutting Conditions Using Tool Wear Prediction Model for End Milling Process. J. Adv. Manuf. Syst. 13, 2014, 41-54.

[Krain, 2007] Krain, H.R., Sharman, A.R.C., Ridgway, K Optimisation of tool life and productivity when end milling Inconel 718TM. Journal of Materials Processing Technology 189, 2007, 153-161.

[Lauro, 2014] Lauro, C.H., Brandão, L.C., Baldo, D., Reis R.A., Davim, J.P. Monitoring and processing signal applied in machining processes - A review. Measurement 58, 2014, 73-86, 14 pp.

[Li, 2017] Li, Z., Wang, G., He, G. Milling tool wear state recognition based on partitioning around medoids (PAM) clustering. Int J Adv Manuf Technol 88, 2017, 1203-1213.

[Lin, 2017] Lin, X., Zhou, B., Zhu, L. Sequential spindle current-based tool condition monitoring with support vector classifier for milling process. Int $\mathrm{J}$ Adv Manuf Technol 92, 2017, 3319-3328, 10 pp.

[M'Saoubi, 2015] M'Saoubi, R., Axinte, D., Soo, S.L., Nobel, C., Attia, H., Kappmeyer, G., Engin, S., Sim, W.-M. High performance cutting of advanced aerospace alloys and composite materials. CIRP Annals 64, 2015, 557-580.

[Muhammad Syafik, 2017] Muhammad Syafik, J., Mohd Razali, M., Syahrul Azwan, S. The Influence of Tool Geometry towards Cutting Performance in Machining Aluminium 7075. MATEC Web Conf. 97, 2017, 1079.

[Niu, 2020] Niu, B., Sun, J., Yang, B. Multisensory based tool wear monitoring for practical applications in milling of titanium alloy. Materials Today: Proceedings 22, 2020, 1209-1217.

[Pfeifer, 2006] Pfeifer, T., Schmitt, R. Autonome Produktionszellen: Komplexe Produktionsprozesse flexibel automatisieren, 2006. Springer, Berlin, 372 pp.

[Rehorn, 2005] Rehorn, A.G., Jiang, J., Orban, P.E. Stateof-the-art methods and results in tool condition monitoring: a review. Int J Adv Manuf Technol 26, 2005, 693-710, 18 pp.

[Sevilla-Camacho, 2011] Sevilla-Camacho, P.Y., HerreraRuiz, G., Robles-Ocampo, J.B., Jáuregui-Correa, J.C Tool breakage detection in CNC high-speed milling based in feed-motor current signals. Int $\mathrm{J}$ Adv Manuf Technol 53, 2011, 1141-1148.

[Shafique, 2014] Shafique, U., Qaiser, H. A Comparative Study of Data Mining Process Models (KDD, CRISP. DM and SEMMA). International Journal of Innovation and Scientific Research 12, 2014, 217-222, 6 pp.

[Suresh Kumar Reddy, 2005] Suresh Kumar Reddy, N., Venkateswara Rao, P. Selection of optimum tool geometry and cutting conditions using a surface roughness prediction model for end milling. Int $\mathrm{J}$ Adv Manuf Technol 26, 2005, 1202-1210.

[Teti, 2010] Teti, R., Jemielniak, K., O’Donnell, G., Dornfeld, D. Advanced monitoring of machining operations. CIRP Annals 59, 2010, 717-739.

[Virtanen, 2020] Virtanen, P., Gommers, R., Oliphant, T.E., Haberland, M., Reddy, T., Cournapeau, D., Burovski, E., Peterson, P., Weckesser, W., Bright, J., van der Walt, Stéfan J., Brett, M., Wilson, J., Millman, K.J., Mayorov, N., Nelson, A.R.J., Jones, E., Kern, R., 
Larson, E., Carey, C.J., Polat, VanderPlas, Jake, Laxalde, D., Perktold, J., Cimrman, R., Henriksen, I., Quintero, E.A., Harris, C.R., Archibald, A.M., Ribeiro, A.H., Pedregosa, F., van Mulbregt, P., SciPy 1.0 Contributors. SciPy 1.0: Fundamental Algorithms for Scientific Computing in Python. Nature Methods 17, 2020, 261-272.

[Wan, 2017] Wan, M., Feng, J., Zhang, W.-H., Yang, Y., $\mathrm{Ma}, \mathrm{Y}$.-C. Working mechanism of helix angle on peak cutting forces together with its design theory for peripheral milling tools. Journal of Materials Processing Technology 249, 2017, 570-580.

[Wang, 2013] Wang, G., Guo, Z., Yang, Y. Force sensor based online tool wear monitoring using distributed Gaussian ARTMAP network. Sensors and Actuators A: Physical 192, 2013, 111-118, 8 pp.

[Westermann, 2015] Westermann, H.-H., Kruse, A., Eisinger, E., Steinhilper, R. Development of an Energy-Efficient Cutting Edge Geometry for Solid End
Mills: A Design of Experiments-Based Approach, 2015.

[Wirth, 2000] Wirth, R., Hipp, J. CRISP-DM: Towards a Standard Process Model for Data Mining. Proceedings of the 4th international conference on the practical applications of knowledge discovery and data mining, 2000. Springer, 29-39, $11 \mathrm{pp}$.

[Wu, 2017] Wu, D., Jennings, C., Terpenny, J., Gao, R.X., Kumara, S. A Comparative Study on Machine Learning Algorithms for Smart Manufacturing: Tool Wear Prediction Using Random Forests. Journal of Manufacturing Science and Engineering 139, 2017, 237.

[Zhu, 2009] Zhu, K., Wong, Y.S., Hong, G.S. Wavelet analysis of sensor signals for tool condition monitoring: A review and some new results. International Journal of Machine Tools and Manufacture 49, 2009, 537-553, 17 pp. 